\title{
Differential mortality drives life-history evolution and population dynamics in the fish Brachyrhaphis rhabdophora
}

Jerald B. Johnson

jerry_johnson@byu.edu

J. Jaime Zuniga-Vega

Follow this and additional works at: https://scholarsarchive.byu.edu/facpub

Part of the Biology Commons

\section{Original Publication Citation}

Johnson, Jerald B., and J. Jaime Zuniga-Vega. "Differential Mortality Drives Life-History Evolution and Population Dynamics in the Fish Brachyrhaphis Rhabdophora." Ecology 9.8 (29): 2243-52.

\section{BYU ScholarsArchive Citation}

Johnson, Jerald B. and Zuniga-Vega, J. Jaime, "Differential mortality drives life-history evolution and population dynamics in the fish Brachyrhaphis rhabdophora" (2009). Faculty Publications. 124. https://scholarsarchive.byu.edu/facpub/124 


\title{
Differential mortality drives life-history evolution and population dynamics in the fish Brachyrhaphis rhabdophora
}

\author{
JERALD B. Johnson ${ }^{1,3}$ AND J. JAIME ZÚÑIGA-VEGA ${ }^{2}$ \\ ${ }^{1}$ Evolutionary Ecology Laboratories, Department of Biology and Monte L. Bean Life Science Museum, \\ Brigham Young University, Provo, Utah 84602 USA
${ }^{2}$ Departamento de Ecología y Recursos Naturales, Facultad de Ciencias, Universidad Nacional Autónoma de México, \\ Ciudad Universitaria, Distrito Federal 04510, México
}

\begin{abstract}
Life-history theory predicts that populations experiencing different levels of extrinsic mortality will evolve divergent reproductive strategies. Previous work in the livebearing fish Brachyrhaphis rhabdophora shows that individuals from populations that occur with piscivorous fish mature earlier and at smaller sizes and have more and smaller offspring than fish from populations without predators. However, until now, there have been no data to demonstrate that differences in mortality rates actually exist between predator and predatorfree sites. Here we present the results of a serial mark-recapture field study designed to estimate mortality rates in natural populations of B. rhabodophora from Costa Rica. We found that fish from predator environments experience higher overall mortality rates and proportionally higher adult mortality rates than fish from predator-free environments. We then ask what impact differences in mortality rates have on B. rhabdophora population dynamics. Using a population matrix modeling approach, we found that $B$. rhabdophora that co-occur with predators have population growth rates similar to those without predators and both have confidence intervals that span $\lambda=1.0$. However, elasticity analysis revealed that the most important life-history stages for population growth in predator environments are found early in life and include growth through early ontogenetic stages and survival as small adults; in contrast, the most important life-history stages for population growth in predator-free environments occur late in life, including survival once large juvenile and adult stages are reached. Hence, we demonstrate two important links between predation and population demography, one ecological due to the direct impacts of predator-induced mortality and the other expressed through predator-mediated reproductive adaptation.
\end{abstract}

Key words: age-specific mortality hypothesis; Brachyrhaphis rhabdophora; Costa Rica; elasticity analysis; live-bearer; Poeciliidae; population growth rate; population matrix modeling; predation.

\section{INTRODUCTION}

Biotic interactions can have a profound impact on both ecological and evolutionary processes. For example, ecological studies of population dynamics frequently focus on the effects that predators have on prey abundance patterns through space and time (Ellis et al. 2007). Similarly, predation is well known as an agent of natural selection. Populations that experience predation often have evolved a suite of adaptations in response to predator-mediated mortality risk. Among the most impressive adaptations to predation are modifications to individual life-history strategies, including evolutionary shifts in the timing of maturation, the amount of energy invested in reproduction, the number and size of offspring produced, and the length of the reproductive life span (Wellborn 1994, Day et al. 2002, Kaliszewicz et al. 2005). Interestingly, these reproductive life-history

Manuscript received 10 October 2007; revised 29 August 2008; accepted 20 November 2008. Corresponding Editor: A. S. Flecker.

${ }^{3}$ E-mail: jerry.johnson@byu.edu traits are also known to shape prey population dynamics. Hence, predation can have a direct impact on population dynamics through predator-mediated mortality and an indirect impact through the evolution of reproductive life-history strategies. Such feedback loops provide an important link between population dynamics and evolutionary adaptation.

Ultimately, the demographic effect of any adaptation must be measured in terms of its contribution to an organism's life history, specifically fecundity, survival, or ontogenetic growth. These "vital rates" determine the rate at which a population can grow (or decline) and underpin the nature of population persistence through time (Williams et al. 2002). Classic matrix projection models (Caswell 2001) provide a life table framework for translating age-specific (or stage-specific) population vital rates into a population growth rate $(\lambda)$. When populations are increasing in size and have a stable age (or stage) distribution, vital rates can also be used to calculate the intrinsic rate of increase $(r=\ln (\lambda))$, a standard measure of the evolutionary fitness of a population (Charlesworth 1994). In a comparative 
framework, the population matrix approach is particularly useful as it provides a way to examine the effects of divergent life-history strategies on variation in population dynamics and fitness within a species.

Given that the link between adaptation and demography must be expressed through fecundity, survival, or growth, we are often interested in knowing which of these vital rates has the greatest impact on population growth and at what life stage (de Kroon et al. 2000). In particular, we want to know whether natural selection has favored the evolution of stage-specific traits that are expected to have the greatest impact on population fitness. Elasticity and sensitivity analyses provide a way to address these questions, especially when comparisons can be made between populations that experience divergent selective regimes. Elasticity is a perturbation measure that quantifies the proportional change in population growth rate as a function of a proportional change in a projection matrix vital rate (e.g., fecundity, survival, or growth) (de Kroon et al. 1986). It can be interpreted as the relative contribution of a vital rate to population growth. Sensitivity is a perturbation measure that quantifies the absolute change in population growth rate that would result from an absolute change in a vital rate (van Tienderen 2000, Caswell 2001). Those vital rates with the highest sensitivity estimates are expected to experience the strongest natural selection (Benton and Grant 1999, Caswell 2001). Moreover, when sufficient genetic variation exists, these traits should undergo the most rapid evolution and should show the greatest level of evolutionary divergence when compared among populations that have evolved in response to divergent selective regimes (Lande 1982, Charlesworth 1994). Despite these clear expectations, empirical studies testing such predictions remain uncommon, especially in natural systems.

The live-bearing fish Brachyrhaphis rhabdophora provides an excellent model to examine the link between adaptive life-history evolution and local population dynamics. Natural populations of this Central American fish show adaptive divergence in several reproductive traits, including timing of maturation and the number and size of offspring produced by females at a given body size (Johnson 2001a, Johnson and Belk 2001). Differences among populations in reproductive rates are strongly associated with divergent selective environments, which are characterized by a tightly correlated suite of ecological factors that include the presence or absence of piscivorous predators (Johnson 2002). Hence, it seems likely that populations with different reproductive adaptations also experience different mortality regimes, a finding well-documented in guppies (Reznick et al. 1996, Rodd and Reznick 1997, Reznick and Bryant 2007). In fact, the observed pattern of life-history divergence among Brachyrhaphis populations, early maturation with many small offspring in predator locations, delayed maturation with few large offspring in predatorfree locations (Johnson and Belk 2001), strongly hints that evolutionary divergence is driven by differential mortality. Given this pattern of life-history divergence, the age-specific mortality hypothesis (Gadgil and Bossert 1970, Schaffer 1974, Reznick et al. 1996) predicts that populations that co-occur with predators should experience high adult mortality and low juvenile mortality relative to populations in predator-free environments. Field estimates of mortality are needed to test the validity of this explanation for life-history divergence in $B$. rhabdophora and simultaneously would provide vital rates of survivorship needed to examine the impact of life-history adaptations on population dynamics.

In this study, we report field estimates of mortality rates and growth in B. rhabdophora derived from serial markrecapture experiments. We combine these data with previous findings on population divergence in fecundity (Johnson and Belk 2001) to answer three fundamental questions. First, are there detectable differences in mortality rates among natural populations of $B$. rhabdophora, and if so, are they consistent with the expectations of the age-specific mortality hypothesis? Second, what are the population dynamic consequences of divergent lifehistory evolution among B. rhabdophora populations? And finally, using elasticity analysis, which vital rates and life cycle paths have the greatest impact on population growth rates and, by extension, on population fitness? We address these questions by comparing mortality, fecundity, and growth in B. rhabdophora from two evolutionarily divergent populations in Costa Rica. Our results show that local adaptive life-history evolution, coupled with differential mortality rates, can have a profound impact on local demographic patterns.

\section{Methods}

\section{Study populations and background}

Brachyrhaphis rhabdophora (Poeciliidae) is a small livebearing fish native to streams and rivers in northwestern Costa Rica (see Plate 1). Populations occupy a wide range of habitats, with the most pronounced ecological differences between habitats characterized by the presence or absence of the predatory fish Parachromis dovii (Cichlidae) (reviewed in Johnson 2002). Here we refer to these habitat types as "predator" and "no-predator" (or "predator-free") environments, but recognize that $P$. dovii may not be the only agent of mortality present in these systems; other kinds of predators, or other ecological factors, could also contribute to B. rhabdophora mortality rates (Johnson 2002). Nonetheless, the presence or absence of $P$. dovii accurately predicts several divergent life-history traits between $B$. rhabdophora populations: populations from predator environments mature at smaller body sizes and have more and smaller offspring than their counterparts from predator-free environments (Johnson and Belk 2001). Common garden experiments demonstrate a genetic basis for these traits, indicating an evolutionary shift has occurred in reproductive strategies between habitats (Johnson 2001a). Moreover, phylogenetic data reveal that this pattern of 
evolutionary divergence has occurred repeatedly in several replicated drainages, supporting the argument that natural selection is responsible (Johnson 2001b). These data all point to differences in mortality as the cause for divergence life-history evolution in this species.

In this study, we estimate mortality rates from two genetically isolated populations of B. rhabdophora found within the Rio Cañas drainage basin in northwestern Costa Rica: Quebrada Grande, a predator-free population, and Rio Javilla, a predator population (locations 27 and 31 in Johnson and Belk [2001]). These locations are characterized by a pool-riffle-pool stream structure, with $B$. rhabdophora individuals occurring predominately in pools. However, riffles are not a barrier to fish movement, even during low-water dry seasons, which meant that it was necessary to estimate mortality and growth rates using a serial mark-recapture design (Appendix A). This logistical constraint allowed us to focus on two populations for this study. Therefore, we selected sites that typify predator and predator-free environments in terms of habitat, stream characteristics, and predator composition (Johnson 2002) and that had fish with reproductive traits that fell within the ranges of populations examined in earlier comparative studies (Johnson and Belk 2001).

\section{Mark-recapture experiment}

To estimate mortality rates and transitional growth rates we used a serial mark-recapture design. The experimental protocol and method for estimating stagespecific mortality rates are described in detail in Appendix A. We used a model selection framework to test for differences in mortality rates among five ontogenetic stages, newborns, small juveniles, large juvenile, small adult, and large adults (defined in Appendix A), within each of the predation environments (Burnham and Anderson 2002; see Fig. 1). We compared four competing mark-recapture models: (1) mortality rates constrained to be identical across all five stages; (2) mortality rates allowed to vary across all five stages; (3) mortality rates for the three nonreproducing stages constrained to be identical and mortality rates for the two adult stages constrained to be identical; and (4) mortality rates for the three nonreproducing stages and small-adult stage constrained to be identical but allowed to vary from the large-adult stage. The latter two models test the idea that newborn/juvenile and adult mortality rates differ from one another or that mortality in the largest adult size class differs from the other four ontogenetic stages. These models can be compared to the two competing models of "no difference among stages" and "all stages differ from one another," models 1 and 2, respectively. We predicted that model 3 or 4 would show the greatest support, as this would indicate a difference between adult and newborn/juvenile mortality rates, consistent with the agespecific mortality hypothesis. Model fit was evaluated using corrected Akaike Information Criterion $\left(\mathrm{AIC}_{\mathrm{c}}\right)$

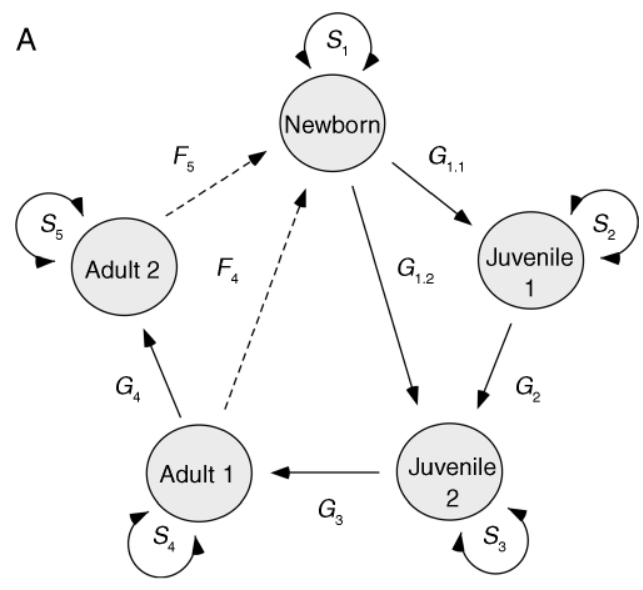

$\mathbf{T}=\left[\begin{array}{ccccc}S_{1} & 0 & 0 & F_{4} & F_{5} \\ G_{1.1} & S_{2} & 0 & 0 & 0 \\ G_{1.2} & G_{2} & S_{3} & 0 & 0 \\ 0 & 0 & G_{3} & S_{4} & 0 \\ 0 & 0 & 0 & G_{4} & S_{5}\end{array}\right]$

FIG. 1. (A) Life cycle graph of the live-bearing fish Brachyrhaphis rhabdophora. Nodes represent five ontogenetic stages that individuals progress through from birth to the end of life (defined in Methods: Mark-recapture experiment). Solid arrows represent transitions from one stage to another or stasis in a particular stage $(G$, survival with progression to a larger size class; $S$, survival staying in the same size class). Dashed arrows represent reproduction $(F$, mean fecundity of females in that stage). Subscripts identify size ontogenetic stages as follows: 1 , newborn; 2 , small juvenile; 3 , large juvenile; 4 , small adult; and 5, large adult. The decimal subscripts on $G_{1.1}$ and $G_{1.2}$ represent the growth of newborn individuals into the smalljuvenile (juvenile 1) and large-juvenile (juvenile 2) stages, respectively. Note that only in the Grande population did newborns grow into juvenile 2 during a single iteration of the cycle. (B) Projection matrix for B. rhabdophora. Terms in the matrix coincide with those in the life cycle graph. The matrix can be interpreted as a numeric depiction of the life cycle graph. Zero values represent cases not observed in our study.

scores, with the lowest score indicating the best-fitting model (Johnson and Omland 2004).

\section{Life cycle graph and projection matrix: combining survival, growth, and fecundity}

We modeled population dynamics in this study using a matrix approach. This required identifying a set of biologically meaningful life stages in B. rhabdophora. We delineated five size classes that correspond to different ontogenetic stages. The matrix modeling approach also required estimates of fecundity and mortality for each of these stages, as well as the rate of transition from one stage to another (due to growth). Finally, it was necessary to establish an appropriate time interval for 
a single iteration of the life cycle model. The interbrood interval for $B$. rhabdophora is approximately 29 days (J. B. Johnson, unpublished data). Hence, our fecundity, mortality, and transition rates for both predator and predator-free populations were calculated over this time period. With these data we constructed a $5 \times 5$ projection matrix (Fig. 1B) that is visually depicted as a life cycle graph (Fig. 1A). The matrix elements $\left(a_{i j}\right)$ (detailed in Caswell 2001) are identified in the matrix as follows: (1) stage-specific fecundity occurs in the first row of the matrix; (2) stage-specific survival for individuals that remain in the same stage (stasis) is found along the main diagonal of the matrix; and (3) survival with progression to larger size classes (growth) is found along the sub-diagonals. Our input values to the matrix for each population in this study were taken from the published life-history data in Johnson and Belk (2001) and from survival estimates calculated here. Stage-specific fecundity was measured as the mean number of embryos per female in each ontogenetic stage. Survival rates for stasis and growth entries were calculated from the stagespecific survival rates obtained from the program MARK (White and Burnham 1999). We partitioned stage-specific survival estimates into "stasis" and "growth" components using the observed proportion of individuals that remained in the same size class over a four-week period vs. those that grew to a larger size class.

\section{Demographic analyses: population growth rates, fitness, elasticities, and loop analysis}

Projection matrices can be used to reveal several demographic patterns. Here we examine three population traits: the finite rate of population increase $(\lambda)$, which is the dominant eigenvalue of the matrix; the stable stage structure $(w)$ taken from the right eigenvector of the matrix; and the distribution of stage-specific reproductive values $(v)$ taken from the left eigenvector of the matrix (Caswell 2001). We tested for differences (using a $\chi^{2}$ test) between the observed proportion of individuals in each size class and the projected proportion of individuals $(w)$ under a stable stage distribution. We also compared the projected stable stage distributions between the predator and predator-free sites (again, using a $\chi^{2}$ test). Combined, these analyses reveal whether there are differences in demographic traits between the predator and no-predator populations. Confidence intervals (95\%) for $\lambda$ values were calculated using the analytical method of Alvarez-Buylla and Slatkin (1991).

We were also interested in identifying which lifehistory traits, which stages, and which demographic pathways have greatest overall impact on population growth rates. To assess the relative contribution to population growth of each element in the matrix, we used elasticity analysis (de Kroon et al. 1986, 2000, Benton and Grant 1999). This first required calculating the sensitivity of population growth rate to perturbation of each element in the matrix; sensitivities $\left(s_{i j}\right)$ measure absolute changes in $\lambda$ that would result from absolute changes in each matrix entry $\left(s_{i j}=\partial \lambda / \partial a_{i j}\right)$. Because matrix elements are measured in different units, it is helpful to standardize sensitivities in order to make comparisons across life-history traits. Elasticities are standardized sensitivities $\left(e_{i j}=s_{i j} \times\left[a_{i j} / \lambda\right]\right)$, which by definition sum to one (Mesterton-Gibbons 1993). Consequently, they can be used to compare among vital rates, stage classes, and populations (de Kroon et al. 2000). In simple terms, this means that the higher the elasticity value, the greater the contribution of that trait to overall population growth rate. Similarly, we can decompose the overall $B$. rhabdophora life cycle into distinct demographic sub-cycles, also known as "loops," and can evaluate their respective elasticities (van Groenendael et al. 1994; described in Appendix B).

To compare elasticities between our study sites or between ontogenetic stages or reproductive loops within each locality, we used a randomization test approach following Caswell (2001). In brief, while preserving the structure of the original samples, individuals were randomly assigned to localities or to different life stages (depending on the comparison of interest), and the difference in elasticity measures between sites (or between ontogenetic stages) was calculated. This procedure was repeated 500 times each for the comparison between habitat types and among stages within habitat types. This resulted in a distribution of differences in elasticity values against which our observed values could be compared. Here we report the calculated elasticity values for the contrast of interest and the probability that these values are statistically identical based on the randomization test (with $P<0.05$ used as our threshold to assign statistical significance).

\section{RESUlts}

\section{Mortality, fecundity, and ontogenetic growth rates}

We found differences in mortality rates between $B$. rhabdophora populations from the predator vs. the nopredator environment (Fig. 2A). Overall, fish that cooccur with the native cichlid predator showed lower rates of survival than their predator-free counterparts (data pooled across stages, $t_{8}=3.305, P=0.011$ ). However, the greatest differences in survival rates occurred in the largest size class (adult 2), with large adults from the predator-free environment having a survival rate of 0.97 compared to large adults from predator environments with a survival rate of only 0.64 . There were also differences in the trends of mortality across ontogenetic stages in the predation environment relative to the no-predator environment (Fig. 2A). In the predator-free habitat, survivorship was relatively constant across the first four ontogenetic stages with a marked increase in the large-adult stage. The best-fitting survivorship model was one in which the large-adult stage was constrained to be different from the remaining four stages (Table 1). In the predator habitat, survival was also relatively constant across the first four 

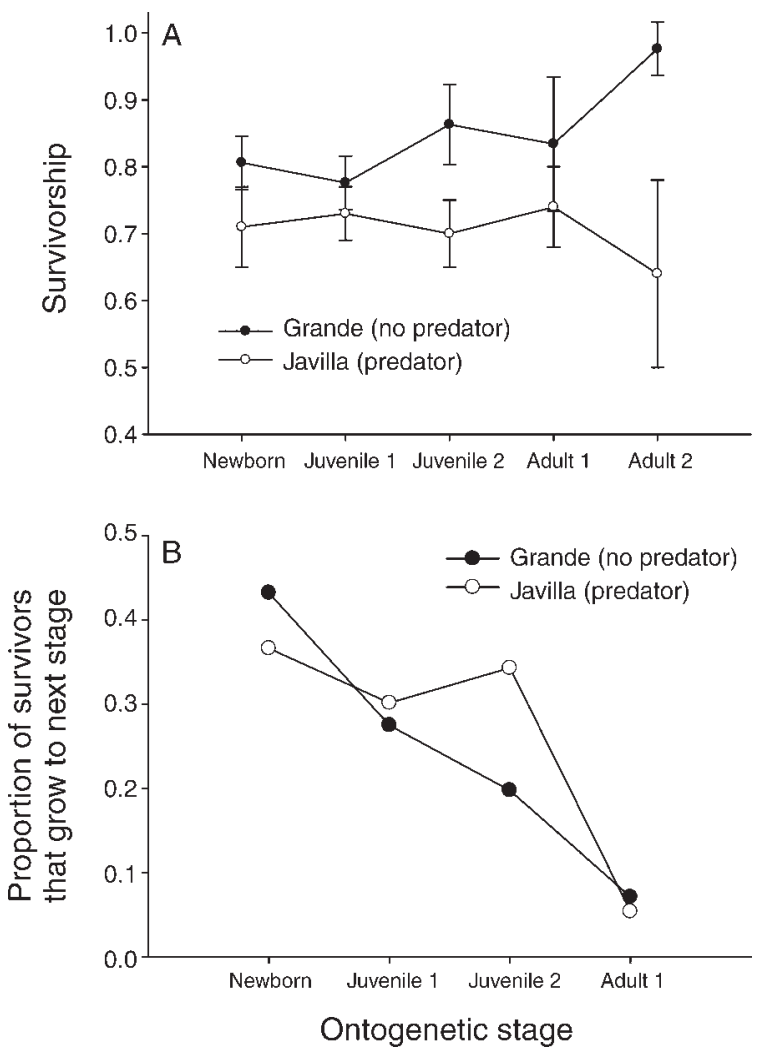

FIG. 2. (A) Survival rates (mean \pm SD) across five ontogenetic stages for both predator and no-predator populations. (B) Comparison of growth from one ontogenetic stage to the next during the four-week mark-recapture period. The study was conducted using two genetically isolated populations of $B$. rhabdophora found within the Rio Cañas drainage basin in northwestern Costa Rica: Quebrada Grande, the no-predator population, and Rio Javilla, the predator population.

ontogenetic stages, but followed by a decrease in survival in the large-adult stage. Again, the best-fitting survivorship model was also one in which the largeadult stage was constrained to be different from the remaining four stages, although the model of identical survival across all five life stages also had strong support (Table 1). Hence, we see an overall pattern of lower survival in the predator population than in the nopredator one, with the greatest difference in mortality rates between the two habitat types found in the size class containing the largest individuals (Fig. 2A).

Stage-specific reproductive rates were notably higher in fish from the predator habitat than fish from the predator-free habitat (Table 2). The results pooled by ontogenetic stages here are consistent with the fecundity measures reported between predator and no-predator populations of B. rhabdophora in Johnson and Belk (2001). In short, fish that co-occur with predators show higher fecundity at both small- and large-adult stages relative to their counterparts from no-predator habitats.

Finally, by individually marking each fish in our study, we were also able to determine the proportion of surviving individuals that grew into larger size classes over the four-week mark-recapture study (corresponding to a single iteration of the matrix model). In both habitat types, individuals in earlier life stages (i.e., newborns and juveniles) were most likely to transition into subsequent ontogenetic stages (Fig. 2B) with $\sim 40 \%$ of newborns advancing relative to $\sim 5 \%$ of adults advancing. The one notable difference between habitat types was that large juveniles from the predator environment were more likely to grow in adulthood than the same stage of fish from the non-predator environment (Fig. 2B).

\section{Population growth rates and elasticities}

Population growth rates did not statistically differ between the predator and predator-free environments, although the point estimate for the predator-free site was higher (Javilla, $\lambda=1.049 \pm 0.198$ vs. Grande, $\lambda=$ $1.182 \pm 0.222$; Table 2 ). Both $\lambda$ values were greater than one, indicating positive population growth; however, the predator site was very close to the zero population growth threshold and the 95\% confidence interval for both estimates spanned 1.0. Neither population conformed to the predicted stable stage distributions

TABLE 1. A comparison of four competing hypotheses of survival in the live-bearing fish Brachyrhaphis rhabdophora, evaluated separately for the (A) no-predator (Grande) and (B) predator (Javilla) populations in Costa Rica.

\begin{tabular}{llrr}
\hline \hline Model & \multicolumn{1}{c}{ Hypothesis } & AIC $_{\mathrm{c}}$ & $\Delta \mathrm{AIC}_{\mathrm{c}}$ \\
\hline A) No-predator & & 1034.84 & \\
4 & different survival between first four life stages and large-adult stage & 1037.15 & 0.0 \\
3 & different survival between juvenile stages and adult stages & 1038.87 & 4.31 \\
2 & different survival across all five life stages & 1045.03 & 10.19 \\
1 & identical survival across all five life stages & \\
B) Predator & & 914.75 \\
4 & different survival between first four life stages and large-adult stage & 915.01 & 0.0 \\
1 & identical survival across all five life stages & 916.99 & 0.27 \\
3 & different survival between juvenile stages and adult stages & 917.71 & 2.24 \\
2 & different survival across all five life stages & 2.96 \\
\hline
\end{tabular}

Notes: Each of the four models represents a different hypothesis (described and numbered in Methods: Mark-recapture experiment). The fit of each model to the observed data was evaluated using the corrected Akaike Information Criterion ( $\mathrm{AIC}_{\mathrm{c}}$ ); the model with the lowest $\mathrm{AIC}_{\mathrm{c}}$ score best fits the data. Models with $\Delta \mathrm{AIC}_{\mathrm{c}}$ values $<2$ are considered to have support comparable to the best model. 
TABLE 2. Population projection matrices and demographic results for populations of Brachyrhaphis rhabdophora from (A) no-predator (Grande) and (B) predator (Javilla) environments.

\begin{tabular}{lccccccr}
\hline \hline Life history stage & Newborn & Juvenile 1 & Juvenile 2 & Adult 1 & Adult 2 & $w$ & $v$ \\
\hline A) No-predator & & & & & & & \\
Newborn & 0.46 & 0 & 0 & 2.70 & 7.90 & 0.50 & 1.0 \\
Juvenile 1 & 0.29 & 0.58 & 0 & 0 & 0 & 0.24 & 1.6 \\
Juvenile 2 & 0.06 & 0.22 & 0.69 & 0 & 0 & 0.17 & 4.3 \\
Adult 1 & 0 & 0 & 0.17 & 0.78 & 0 & 0.07 & 12.6 \\
Adult 2 & 0 & 0 & 0 & 0.06 & 0.98 & 0.02 & 39.2 \\
q & 0.19 & 0.20 & 0.14 & 0.16 & 0.02 & & \\
B) Predator & & & & & & & \\
Newborn & 0.45 & 0 & 0 & 4.00 & 8.60 & 0.56 & 1.0 \\
Juvenile 1 & 0.26 & 0.51 & 0 & 0 & 0 & 0.27 & 2.3 \\
Juvenile 2 & 0 & 0.22 & 0.46 & 0 & 0 & 0.10 & 5.6 \\
Adult 1 & 0 & 0 & 0.24 & 0.70 & 0 & 0.07 & 13.9 \\
Adult 2 & 0 & 0 & 0 & 0.04 & 0.64 & 0.01 & 21.0 \\
qx & 0.29 & 0.27 & 0.30 & 0.26 & 0.36 & & \\
\hline
\end{tabular}

Notes: Population growth rates are: no-predator, $\lambda=1.182 \pm 0.222$; predator, $\lambda=1.049 \pm$ 0.198 (mean $\pm 95 \% \mathrm{CI}$ ). Abbreviations are: $q_{x}$, estimates of mortality rates in each life stage; $w$, the projected stable stage distribution; and $v$, stage-specific reproductive values. Lifehistory stages are defined in Methods: Mark-recapture experiment and explained in detail in Appendix A.

expected from the population projection matrices (Javilla, $\chi_{4}^{2}=304.35, P<0.001$; Grande, $\chi_{4}^{2}=137.64$, $P<0.001)$. Moreover, the observed distribution of individuals in each ontogenetic stage also differed between the two habitat types $\left(\chi_{4}^{2}=295.03, P<\right.$ 0.001 ), with fewer large-adult individuals found at the predator site than in the no-predator site. Finally, we found differences between the two sets of projected stable stage distributions between habitat types $\left(\chi_{4}^{2}=\right.$ 48.77, $P<0.001)$ : the predator matrix predicted a higher proportion of individuals in the newborn and smalljuvenile size classes compared to the non-predator matrix and a lower proportion of individuals in the large-juvenile and large-adult size classes. Differences in mortality rates between habitat types also resulted in a much higher reproductive value for large adults in the non-predator environment relative to those in the predator environment (Table 2; vector of $v$ values).
Elasticity estimates revealed clear differences between the predator and no-predator populations in terms of which transition probabilities and vital rates most affected population growth rates. In the predator-free population, the vital rates that contributed most to population growth were those associated with surviving and staying in same size class for large juveniles and adults (Table 3A). Survival and stasis in the small-adult size class was also important in the predator site (Table 3B). However, unlike the predator-free population, most of important life stages were in the newborn and juvenile stages, and these included survival and growth to subsequent developmental stages (Table 3B). Simply put, growing and surviving to the smallest (and safest) reproductive stage was paramount for individuals in the predator environment, whereas persisting in the larger size classes mattered most in the predator-free environment where adult mortality rates were much lower.

TABLE 3. Elasticity matrices for populations of Brachyrhaphis rhabdophora from (A) nopredator (Grande) and (B) predator (Javilla) environments.

\begin{tabular}{lccccc}
\hline \hline Life-history stage & Newborn & Juvenile 1 & Juvenile 2 & Adult 1 & Adult 2 \\
\hline A) No-predator & & & & & \\
Newborn & 0.058 & 0 & 0 & 0.049 & 0.042 \\
Juvenile 1 & 0.058 & 0.056 & 0 & 0 & 0 \\
Juvenile 2 & 0.033 & 0.058 & $\mathbf{0 . 1 2 8}$ & 0 & 0 \\
Adult 1 & 0 & 0 & 0.091 & $\mathbf{0 . 1 7 7}$ & 0 \\
Adult 2 & 0 & 0 & 0 & 0.042 & $\mathbf{0 . 2 0 6}$ \\
\& elasticities & 0.149 & 0.114 & 0.219 & 0.268 & 0.248 \\
B) Predator & & & & & \\
Newborn & 0.084 & 0 & 0 & 0.093 & 0.019 \\
Juvenile 1 & $\mathbf{0 . 1 1 2}$ & $\mathbf{0 . 1 0 6}$ & 0 & 0 & 0 \\
Juvenile 2 & 0 & $\mathbf{0 . 1 1 2}$ & 0.087 & 0 & 0 \\
Adult 1 & 0 & 0 & $\mathbf{0 . 1 1 2}$ & $\mathbf{0 . 2 2 5}$ & 0 \\
Adult 2 & 0 & 0 & 0 & 0.019 & 0.030 \\
E elasticities & 0.196 & 0.218 & 0.199 & 0.337 & 0.049 \\
\hline
\end{tabular}

Note: Elasticity values $>0.100$ are shown in bold type. 
Given that elasticity values are additive, we can also report which life stages and which demographic processes contribute most to population growth rates. In the predator-free habitat, the newborn and juvenile stages have a relative contribution to population growth that is comparable to that of the two adult stages (summed elasticities of 0.482 vs. $0.516, P=0.06$; Table $3 \mathrm{~A}$ ). The contribution of life stages in the predator habitat shows a somewhat different trend. Newborn and juvenile stages are more important than their no-predator counterparts (summed elasticity $=0.613$ vs. $0.386, P<0.001$ ). However, the most substantial difference is that in the predator habitat, the small-adult stage is much more important than the large-adult stage to overall population growth rates $(0.337$ vs. $0.049, P<0.001$; Table $3 \mathrm{~B})$. In the non-predator habitat, the relative contribution of each adult stage is similar ( small adults $=0.268$, large adults $=0.248, P=0.24)$. When comparing the relative importance of fecundity, survival and advancement to the next stage (growth) or survival and persistence in the same stage (stasis) to overall population growth, we found that the two types of habitats showed the same pattern (Fig. 3A). In both habitat types, survival mattered more than fecundity, with persistence in a given ontogenetic stage being more important to population growth than graduation to subsequent ontogenetic stages (Fig. 3).

\section{Discussion}

In this study we set out to address three basic problems. First, we wanted to know whether differences in mortality rates could explain observed differences in life-history traits between $B$. rhabdophora populations that occur with fish predators vs. those that exist without fish predators (Johnson and Belk 2001, Johnson 2002). It appears quite clearly that they can: high mortality rates in the large-adult size class that typifies the predator environment predicts decreased size at maturity, increased number of offspring, and decreased size of offspring; the opposite patterns occur in the predatorfree environment. Second, against this backdrop of differential mortality, we wanted to explore the population dynamic and fitness consequences of divergent lifehistory evolution among $B$. rhabdophora populations. In other words, we wanted to know what kind of effect predator-mediated life-history evolution and differential mortality have had on population growth rates. Finally, we wanted to determine which vital rates, which life stages, and which reproductive pathways have the greatest influence on population growth in B. rhabdophora. We consider each of these problems in turn.

\section{Age-specific mortality hypothesis}

One of the most explicit sets of predictions that come out of life-history theory is that differences in age- or stage-specific mortality rates among populations should result in divergent life-history evolution (Stearns 1992, Roff 2002). Early theoretical work predicted that high
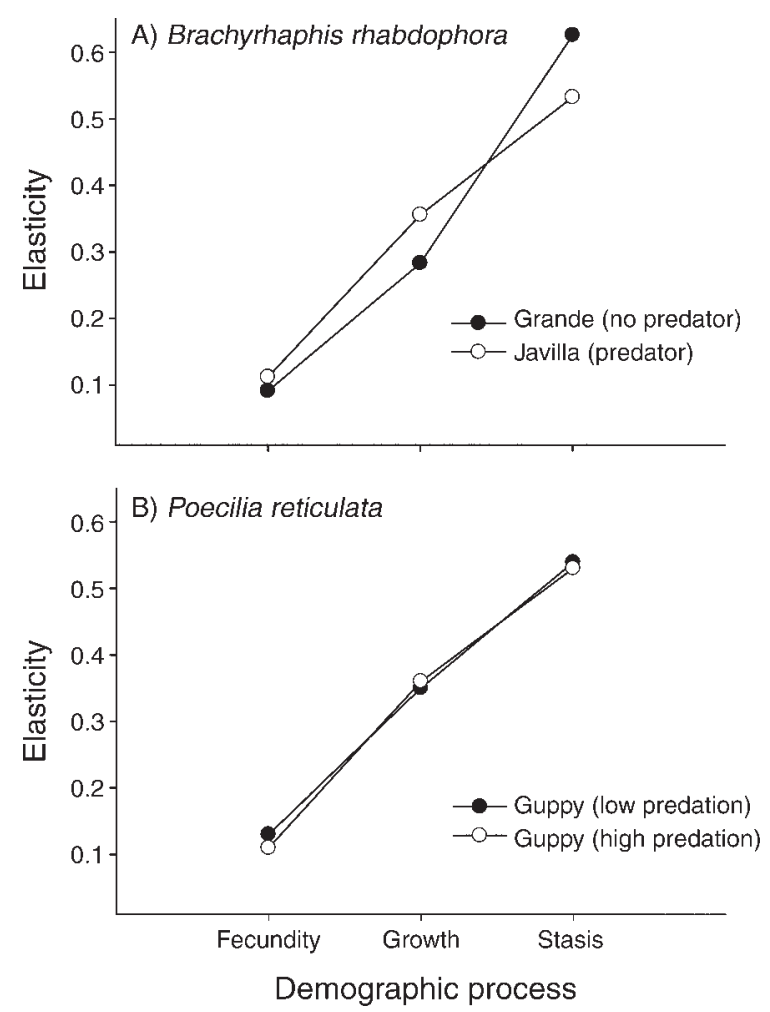

FIG. 3. Elasticity values for the three demographic processes that contribute to population growth rate: fecundity; survival and graduation to a subsequent ontogenetic stage (growth); and survival and persistence in the same ontogenetic stage (stasis). We present results for: (A) Brachyrhaphis rhabdophora from predator and no-predator environments (this study); and (B) guppies from high- and low-predation environments (data from Bronikowski et al. [2002]).

adult mortality relative to juvenile mortality would result in the evolution of earlier age at maturity (and maturity at smaller body sizes if size and ontogenetic stage are related), the production of more and smaller offspring, and increased reproductive effort (Gadgil and Bossert 1970, Law 1979, Michod 1979). More recent theoretical work demonstrates that mortality need not be age-specific, but that overall differences in mortality rates across all ontogenetic stages can have the same effects on life-history evolution (Kozlowski and Uchmanski 1987, Abrams and Rowe 1996, Reznick et al. 1996). Collectively, this body of theory has come to be known as the "age-specific mortality hypothesis," and it underpins some of our most important examples of lifehistory evolution in the wild (Reznick et al. 1990, Wellborn 1994).

Consistent with predictions from the age-specific mortality hypothesis, both overall mortality rate and adult mortality rates are higher in our Rio Javilla (predator) population than in the Quebrada Grande (no-predator) population (Fig. 2A). It appears that at Rio Javilla predators prefer the largest $B$. rhabdophora individuals with almost $36 \%$ mortality rate in this large- 


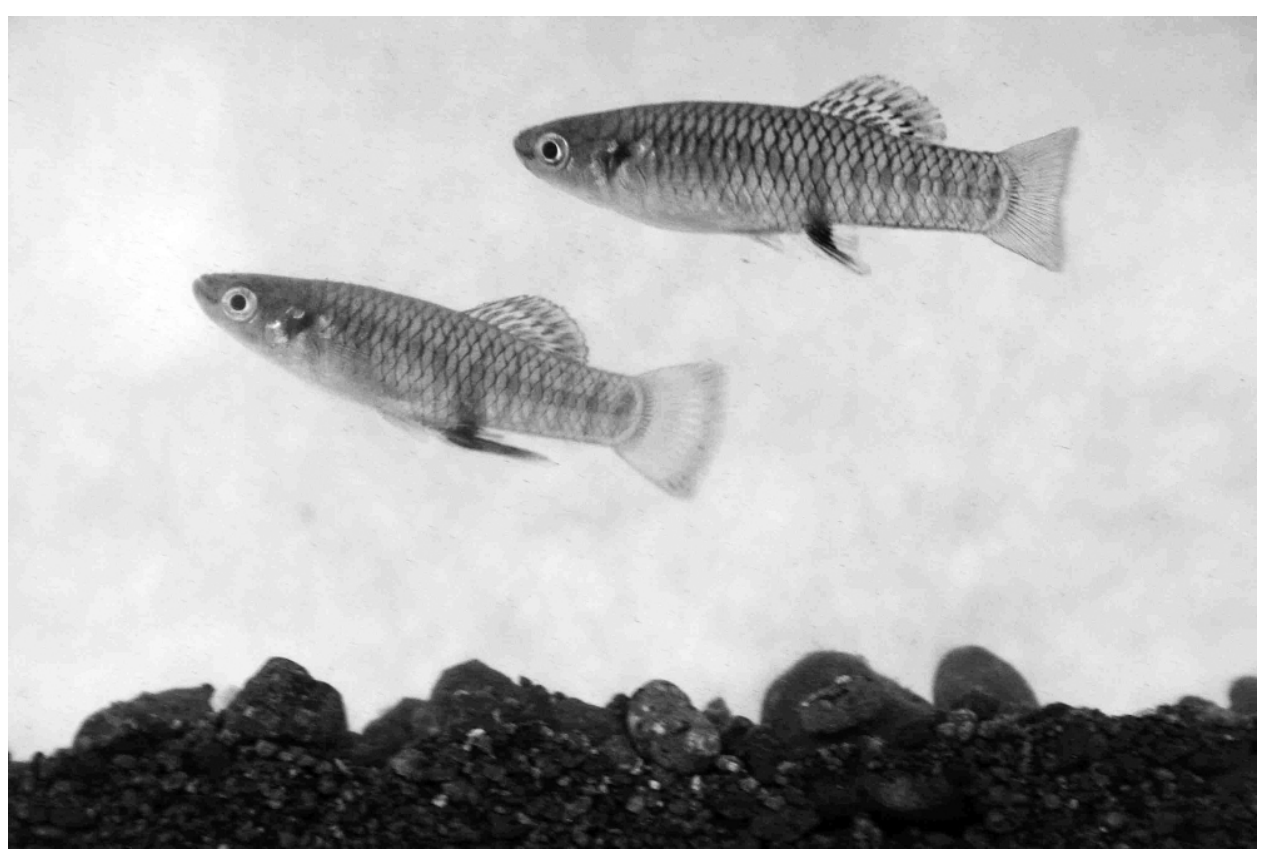

Plate 1. Photograph of two individuals of the live-bearing fish Brachyrhaphis rhabdophora. The male is below, and the female is above. Photo credit: J. B. Johnson.

adult size class (measured over one 28-day reproductive iteration) compared to $<3 \%$ mortality in this same size class from Quebrada Grande. In addition, the mean rate of survival was lower for every ontogenetic stage, from juveniles to large adults, in the predator environment relative to the predator-free environment (Fig. 2A). In short, the pattern of mortality observed here is completely consistent with both sets of theoretical models that predict divergent life-history evolution.

In early work exploring life history variation in $B$. rhabdophora, Johnson (2002) noted that "predation environment" could be decomposed into several component parts. In addition to differences in extrinsic mortality rates, other ecological factors can vary among sites and some of these could also contribute to observed differences in B. rhabdophora life histories. These factors include variation in relative density, variation in canopy cover (which could be linked to resource availability), and differences in stream characteristics such as stream gradient, width, and depth. Although we recognize that these factors could contribute to life-history variation among populations in the wild, differences in extrinsic mortality rates documented here now clearly point to this factor as an important causal agent of life-history evolution in B. rhabdophora.

\section{Impact of life-history evolution on demography and fitness}

A basic problem in population ecology is to understand how variable life histories and differential mortality translate into demographic behavior, including projected population growth and fitness (Caswell 2001, Metcalf and Pavard 2007). Brachyrhaphis rhabdophora popula- tions that experience differences in mortality rates have evolved divergent reproductive strategies (Johnson 2001a, Johnson and Belk 2001). Under such circumstances, the key demographic question is whether or not these variable life-history traits are in fact adaptive, that is, are population growth rates comparable across populations despite variation in the vital rates that make up fitness (Caswell 2001)? We find that fish living in the high-mortality predation environment do not suffer a population fitness cost relative to fish in the predator-free environment; there is no statistical difference in population growth rates between the two kinds of predator environments (Table 2, 95\% CIs overlap). In the presence of predators, B. rhabdophora achieve comparable population growths rates by maturing earlier and shifting reproduction to smaller adult size classes in which the probability of survival is much higher. In the absence of predators, reproduction is delayed and is spread more evenly between both adult size classes. Finally, although there may be differences in density between the two habitat types (Johnson 2002), simulations using our transition matrices suggest that density effects are not likely to render our two population growth rates statistically different (Appendix C).

We found differences in elasticities between the predator and predator-free selective environments. In the predator-free environment only three matrix elements had elasticities greater than 0.10 : survival and stasis in the large-juvenile and the two adult size classes. In contrast, in the predator environment rapid growth and transition from newborn, small-juvenile, and largejuvenile stages to subsequent life stages all had 
elasticities greater than 0.10; and the vital rate with the highest elasticity in the predator environment was survival and stasis in the small-adult stage (0.23). This pattern makes sense; in predator environments overall mortality rates are higher than in predator-free environments and large adults suffer disproportionately higher mortality rates (Fig. 2). Hence, individuals should grow rapidly to the small-adult size class and then should persist in this size class as long as possible to reproduce. It is interesting to note that in the predator environment the lowest elasticity of all vital rates is for survival and stasis of large adults. In short, too few of these individuals survive to have much of an impact on overall population growth rates.

\section{Comparison with guppies}

Similar demographic analyses have been conducted on guppies from Trinidad that occur in high- vs. lowpredation environments (Rodd and Reznick 1997, Bronikowski et al. 2002). Guppies and B. rhabdophora are both live-bearing fishes in the family Poeciliidae, but come from different phylogenetic clades within this family (Hrbek et al. 2007). Yet the two species show a remarkable pattern of evolutionary convergence in terms of divergent life-history evolution in response to predation (Johnson and Belk 2001). In fact, the two species are so similar in their pattern of life-history divergence that discriminant function data taken from guppy life-history traits (Reznick and Endler 1982) can actually be used to predict with complete accuracy whether a $B$. rhabdophora population comes from a predator or a predator-free environment (Johnson and Belk 2001). Given this high degree of evolutionary convergence, we wanted to explore whether these two species also showed similar patterns of demographic convergence.

The most pronounced similarities between B. rhabdophora and guppies are the elasticity values for overall fecundity, growth, and stasis. In our study we found that in both predator and non-predator habitats, fecundity had the lowest elasticity, growth had an intermediate elasticity, and stasis had the highest elasticity (Fig. 3A). Bronikowski et al. (2002) reported the same pattern for guppies in both high- and low-predation environments (Fig. 3B). Such consistency across species and predation environments suggests a general phenomenon: survival (stasis) is clearly more important than fecundity or growth in terms of impact on population fitness.

The summed elasticity values help answer a fundamental question that we raised in our introduction: what is the relative importance of predation in terms of its direct impact on population dynamics through predatormediated mortality vs. the indirect impact of predation through the evolution of life-history strategies? Here, direct effects of predation are manifest by the summed elasticity for stasis, in that this value reveals the impact that survivorship has on population growth rate. For both guppies and $B$. rhabdophora this value is relatively large ( 0.53 and 0.58 , respectively, averaged over predation environments). The indirect effect of predators is manifest through life-history adaptations, which are captured by the summed elasticity for fecundity. These fecundity values are relatively small in both guppies and B. rhabdophora $(0.12$ and 0.10 , respectively, averaged over predation environments). Hence, in both guppies and B. rhabdophora, predators have a much stronger direct effect through mortality on population growth rates than an indirect effect through adaptive life-history evolution.

\section{Conclusion}

Focusing on demographic patterns in B. rhabdophora offers insight into the link between predator-mediated life-history evolution and population dynamics. Differential mortality appears to have shaped a variety of reproductive traits in $B$. rhabdophora, including sizespecific fecundity and the timing of maturation. These traits are important components of the projection matrix used to estimate demographic patterns. Mortality rates also have a direct effect in the population matrix. Hence, our study highlights the basic interaction between predation as an agent of natural selection that drives reproductive evolution and predation as an agent of mortality that directly impacts demography. When combined in a population matrix framework, we see how local life-history evolution leads to variation in demographic trends in nature, an important goal of any research program in population ecology.

\section{ACKNOWLEDGMents}

We thank K. Sachs, A. Theriault, and M. Baker for excellent help with field mark-recapture experiments and members of the Zúñiga-Vega laboratory who helped execute the randomization tests. Petty thieves Tilapia and Brinca Brinca taught us an important lesson about where not to keep your field notes. We are especially grateful to the Álvarez family who has repeatedly provided access to their property outside of Tilarán, Costa Rica; we dedicate this paper to the memory of Edwin Álvarez. The Bakal Family Foundation, the Roger and Victoria Sant Endowment, and Brigham Young University provided funding for this project.

\section{Literature Cited}

Abrams, P. A., and L. Rowe. 1996. The effects of predation on the age and size of maturity of prey. Evolution 50:1052-1064. Alvarez-Buylla, E. R., and M. Slatkin. 1991. Finding confidence limits on population growth rates. Trends in Ecology and Evolution 6:221-224.

Benton, T. G., and A. Grant. 1999. Elasticity analysis as an important tool in evolutionary and population ecology. Trends in Ecology and Evolution 14:467-471.

Bronikowski, A. M., M. E. Clark, F. H. Rodd, and D. N. Reznick. 2002. Population-dynamic consequences of predator-induced life history variation in the guppy. Ecology 83: 2194-2204.

Burnham, K. P., and D. R. Anderson. 2002. Model selection and multimodel inference: a practical information-theoretic approach. Springer, New York, New York, USA.

Caswell, H. 2001. Matrix population models: construction, analysis, and interpretation. Sinauer, Sunderland, Massachusetts, USA. 
Charlesworth, B. 1994. Evolution in age-structured populations. Second edition. Cambridge University Press, Cambridge, UK.

Day, T., P. A. Abrams, and J. M. Chase. 2002. The role of sizespecific predation in the evolution and diversification of prey life histories. Evolution 56:877-887.

de Kroon, H., A. Plaisier, J. M. van Groenendael, and H. Caswell. 1986. Elasticity: the relative contribution of demographic parameters to population growth rate. Ecology 67:1427-1431.

de Kroon, H., J. M. van Groenendael, and J. Ehrlén. 2000. Elasticities: a review of methods and model limitations. Ecology 81:607-618.

Ellis, J. C., M. J. Shulman, M. Wood, J. D. Witman, and S. Lozyniak. 2007. Regulation of intertidal food webs by avian predators on New England rocky shores. Ecology 88:853863.

Gadgil, M., and W. H. Bossert. 1970. Life historical consequences of natural selection. American Naturalist 104:1-24.

Hrbek, T., J. Seckinger, and A. Meyer. 2007. A phylogenetic and biogeographical perspective on the evolution of poeciliid fishes. Molecular Phylogenetics and Evolution 43:986-998.

Johnson, J. B. 2001a. Adaptive life history evolution in the livebearing fish Brachyrhaphis rhabdophora: genetic basis for parallel divergence in age and size at maturity and a test of predator-induced phenotypic plasticity. Evolution 55:14861491.

Johnson, J. B. 2001b. Hierarchical organization of genetic variation in the Costa Rican livebearing fish Brachyrhaphis rhabdophora (Poeciliidae). Biological Journal of the Linnean Society 72:519-527.

Johnson, J. B. 2002. Divergent life histories among populations of the fish Brachyrhaphis rhabdophora: detecting putative agents of selection by candidate model analysis. Oikos 96:8291.

Johnson, J. B., and M. C. Belk. 2001. Predation environment predicts divergent life-history phenotypes among populations of the livebearing fish Brachyrhaphis rhabdophora. Oecologia 126:142-149.

Johnson, J. B., and K. S. Omland. 2004. Model selection in ecology and evolution. Trends in Ecology and Evolution 19: 101-108.

Kaliszewicz, A., K. Johst, V. Grimm, and J. Uchmański. 2005. Predation effects on the evolution of life-history traits in a clonal oligochaete. American Naturalist 166:409-417.

Kozlowski, J., and J. Uchmański. 1987. Optimal individual growth and reproduction in perennial species with indeterminate growth. Evolutionary Ecology 1:214-230.

Lande, R. 1982. A quantitative genetic theory of life history evolution. Ecology 63:607-615.
Law, R. 1979. Optimal life histories under age-specific predation. American Naturalist 114:399-417.

Mesterton-Gibbons, M. 1993. Why demographic elasticities sum to one: a postscript to de Kroon et al. Ecology 74:24672468.

Metcalf, C. J. E., and S. Pavard. 2007. Why evolutionary biologists should be demographers. Trends in Ecology and Evolution 22:205-212.

Michod, R. E. 1979. Evolution of life histories in response to agespecific mortality factors. American Naturalist 113:531-550.

Reznick, D. N., and M. Bryant. 2007. Comparative long-term mark-recapture studies of guppies (Poecilia reticulata): differences among high and low predation localities in growth and survival. Annales Zoologici Fenicci 44:152-160.

Reznick, D. N., H. Bryga, and J. A. Endler. 1990. Experimentally induced life-history evolution in a natural population. Nature 346:357-359.

Reznick, D. N., M. J. Butler IV, F. H. Rodd, and P. Ross. 1996. Life history evolution in guppies (Poecilia reticulata). 6. Differential mortality as a mechanism for natural selection. Evolution 50:1651-1660.

Reznick, D. N., and J. A. Endler. 1982. The impact of predation on life history evolution in Trinidadian guppies (Poecilia reticulata). Evolution 36:160-177.

Rodd, F. H., and D. N. Reznick. 1997. Variation in the demography of guppy populations: the importance of predation and life histories. Ecology 78:405-418.

Roff, D. A. 2002. Life history evolution. Sinauer, Sunderland, Massachusetts, USA.

Schaffer, W. M. 1974. Selection for optimal life histories: the effects of age structure. Ecology 55:291-303.

Stearns, S. C. 1992. The evolution of life histories. Oxford University Press, New York, New York, USA.

van Groenendael, J., H. de Kroon, S. Kalisz, and S. Tuljapurkar. 1994. Loop analysis: evaluating life history pathways in population projection matrices. Ecology 75: $2410-2415$.

van Tienderen, P. H. 2000. Elasticities and the link between demographic and evolutionary dynamics. Ecology 81:666679.

Wellborn, G. A. 1994. Size-biased predation and prey life histories: a comparative study of freshwater amphipod populations. Ecology 75:2104-2117.

White, G. C., and K. P. Burnham. 1999. Program MARK: survival estimation from populations of marked animals. Bird Study 46(Supplement):120-138.

Williams, B. K., J. D. Nichols, and M. J. Conroy. 2002. Analysis and management of animal populations. Academic Press, San Diego, California, USA.

\section{APPENDIX A}

Description of mark-recapture methods used to estimate mortality rates in Brachyrhaphis rhabdophora populations (Ecological Archives E090-156-A1).

\section{APPENDIX B}

Description of the demographic loop analysis in Brachyrhaphis rhabdophora (Ecological Archives E090-156-A2).

\section{APPENDIX C}

Simulation of the effect of density on population growth rates in Brachyrhaphis rhabdophora populations (Ecological Archives E090-156-A3). 